\title{
Influência de diferentes sistemas de preparo do solo nas propriedades físicas de um Latossolo Vermelho distrófico
}

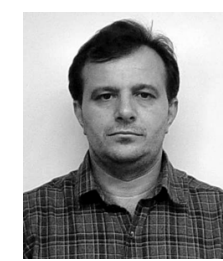

\author{
Cássio A. Tormena ${ }^{1,3}$, Pedro S. Vidigal Filho ${ }^{1,3}$, Antônio C. A. Gonçalves ${ }^{1}$, Marcelo A. Araújo² \& José C. Pintro \\ 1 Departamento de Agronomia da Universidade Estadual de Maringá, PR. Av. Colombo 5790, Campus, CEP 87020-900, Maringá, \\ PR. E-mail: catormena@uem.br (Foto) \\ 2 Doutorando do Programa de Pós-Graduação em Agronomia da Universidade Estadual de Maringá \\ ${ }^{3}$ Bolsista do CNPq
}

Protocolo 189 - 10/12/2002 - Aprovado em 16/12/2003

\begin{abstract}
Resumo: Em geral, os sistemas de preparo do solo têm, como objetivo, oferecer condições físicas adequadas para a otimização do crescimento, desenvolvimento e produtividade das culturas. O propósito deste estudo foi avaliar o efeito dos sistemas de preparo do solo em algumas propriedades físicas de um Latossolo Vermelho distrófico, no Estado do Paraná, cultivado com mandioca. Para isto, utilizaram-se, neste trabalho, os seguintes tratamentos: Plantio Direto - PD (sem revolvimento do solo ou revolvimento apenas ao longo das linhas de plantio); Preparo Mínimo - PM (escarificação a 0,30 m seguido de gradagem niveladora) e Preparo Convencional - PC (aração com arado de aiveca a 0,25-0,30 m de profundidade, seguido de gradagem niveladora). As avaliações feitas nas camadas de 0-0,15 e 0,15-0,30 m constaram das seguintes propriedades físicas do solo: densidade do solo (Ds), volume de macroporos drenados na tensão de 0,006 MPa (Mac), volume de microporos (Mic) e porosidade total (Pt). Avaliou-se, também, a resistência do solo à penetração das raízes (RP) a cada $0,05 \mathrm{~m}$, até a profundidade de $0,40 \mathrm{~m}$, em duas diferentes épocas, em que os resultados indicaram maiores valores de Ds e menores de Mac no PD e no PM em comparação com o PC. No PM e PD constatou-se que o volume de poros com ar na tensão de $0,006 \mathrm{MPa}$ foi menor que $10 \%$ e a RP foi maior no PD em comparação com os demais tratamentos, atingindo valores superiores a $2,0 \mathrm{MPa}$, até a profundidade de $0,25 \mathrm{~m}$. Os resultados sugerem que, nas condições em que o estudo foi conduzido, a qualidade física do solo mostrou-se restritiva no PD devido aos excessivos valores de RP e aos reduzidos valores de porosidade de aeração.
\end{abstract}

Palavras-chave: preparo convencional, plantio direto, resistência do solo á penetração

\section{Influence of different tillage systems in physical properties of a distrophic Red Latosol}

\begin{abstract}
The tillage systems have the objective to offer an adequate physical conditions in soil for growth, development and productivity of crops. The objective of this study was to evaluate the effect of different soil tillage systems on some physical properties of a distrophic Red Latosol soil (Typic Haplorthox), cultivated with cassava, in the Paraná state, Brazil. The treatments were: No-tillage (the soil was just revolved along the planting lines) - NT; Minimum Tillage (subsoiling at $0.30 \mathrm{~m}$ depth, followed by leveling) - MT; and Conventional Tillage (plowing with moldboard plow to the 0.25-0.30 m depth, followed by leveling) - CT. In the layers of 0-0.15 and 0.15-0.30 m, soil physical properties i.e soil bulk density (Bd), macroporosity (Mac), microporosity (Mic) and total porosity (Pt) were evaluated. The soil resistance to root penetration (SRP) was evaluated in $0.05 \mathrm{~m}$ intervals until the depth of $0.40 \mathrm{~m}$, in two different periods of cassava growth. The results indicated higher values of $\mathrm{Bd}$ and lower values of Mac and Pt in NT and MT than in CT system. The air-filled porosity was $<10 \%$ in MT and NT. The SRP was higher in NT than in CT and MT, with values $>2.0 \mathrm{MPa}$ until the depth of $0.25 \mathrm{~m}$. The results of this study suggest that soil physical quality may be restrictive to growth of crops in NT. It may be justified by the higher values of SRP and reduced soil aeration.
\end{abstract}

Key words: conventional tillage, no-tillage, soil resistance to root penetration 


\section{INTRODUÇÃO}

Os diferentes sistemas de preparo do solo têm, como objetivo, oferecer as condições físicas adequadas para o crescimento, desenvolvimento e produtividade das culturas. No entanto, dependendo do solo, do clima e da cultura, os sistemas de preparo podem promover a degradação da qualidade física do solo, com reflexos ambientais e na produtividade das culturas. Em solos de textura superficial média-arenosa e com baixos teores de matéria orgânica, o cultivo sucessivo e o revolvimento excessivo os predispõem às altas taxas de erosão, de compactação e de perdas de matéria orgânica, resultando na degradação física, química e biológica dos mesmos (Cardoso et al., 1992; Fidalski, 1997). Neste contexto estão enquadrados os solos derivados do arenito Caiuá, que ocorrem na região noroeste do estado do Paraná (Carvalho et al., 1994).

A mandioca (Manihot esculenta Crantz) é freqüentemente cultivada em solos de textura superficial média a arenosa, com baixos teores de nutrientes e de matéria orgânica. Segundo Groxko (1997), no Estado do Paraná, a cultura da mandioca ocupa uma área de 156.000 ha, proporcionando uma produção anual de aproximadamente 3,5 milhões de toneladas de raízes tuberosas e a maior parte da área cultivada localiza-se nos solos derivados do Arenito Caiuá, na região noroeste do estado. Souza (1986) afirma que, dentre os diferentes fatores que interferem na determinação da produtividade da cultura da mandioca destaca-se o sistema de preparo do solo. Esta cultura apresenta um ciclo que varia de 10 a 18 meses, de modo que a variação temporal de umidade do solo pode proporcionar condições físicas, desde altamente favoráveis até impeditivas para a cultura, as quais são dependentes da qualidade estrutural determinada pelos sistemas de preparo utilizados. Desta forma, num solo mais compactado ou de baixa estabilidade estrutural, aumenta a probabilidade da cultura ser exposta a algum tipo de estresse físico (Kay, 1990).

Na região noroeste do Paraná, o sistema de preparo do solo mais comumente utilizado no cultivo da mandioca é o preparo convencional (aração + gradagem) devido, principalmente, à disponibilidade de equipamentos para os produtores e pela tradição na utilização deste sistema. Porém, os solos desta região se apresentam altamente suscetíveis à erosão, o que tem estimulado a redução na intensidade de preparo e a manutenção da cobertura do solo pelos resíduos culturais, as quais são estratégias eficientes para o controle das perdas de solo, para a redução das perdas de matéria orgânica e para a manutenção da umidade do solo. Segundo Oliveira et al. (2001), nesta região, sistemas de preparo com redução na movimentação do solo e manutenção de resíduos na superfície, vêm-se tornando alternativas tecnicamente viáveis e desejáveis para a cultura da mandioca, em termos econômicos e conservacionistas.

A adoção de sistemas de preparo com menor revolvimento do solo resulta numa compactação maior nas camadas superficiais dos solos. Ao mesmo tempo, tem-se constatado que culturas de raízes tuberosas são sensíveis à compactação do solo, devido às menores taxas de difusão de oxigênio no solo e pela excessiva resistência à penetração nos solos compactados. A hipótese de que essas alterações nas propriedades físicas podem induzir condições adversas para o desenvolvimento das plantas tem sido confirmada. Por outro lado, estudos sugerem que condições físicas adversas resultam na emissão de sinais hormonais que são enviados da raiz à parte aérea, com reflexos na taxa fotossintética e, por conseqüência, no crescimento e desenvolvimento das plantas (Davies \& Zhang, 1991). Para a cultura da mandioca, o impacto dos sistemas de preparo em indicadores da sua qualidade física e química do solo tem refletido no crescimento e na produtividade da cultura (Howeler et al., 1993; Cadavid et al., 1998).

A densidade e a porosidade do solo refletem o impacto dos estresses aplicados ao solo pelos sistemas de preparo e pelo tráfego de máquinas na área (Kay \& Angers, 2000). Portanto, diferentes operações de preparo podem alterar as propriedades físicas do solo, tais como a densidade do solo (Ds), a porosidade $(\mathrm{Pt})$ e a resistência do solo à penetração das raízes (RP). Estas alterações podem afetar a distribuição, a quantidade e a morfologia das raízes, com reflexos no crescimento da parte aérea das plantas (Klepker \& Anghinoni, 1995). A Ds e a Pt são propriedades freqüentemente utilizadas para caracterizar os efeitos dos sistemas de preparo sobre a estrutura e as propriedades físicas dos solos ( Klepker \& Anghinoni, 1995).

Diferentes estudos têm demonstrado que a compactação do solo resulta num aumento da RP (Tormena \& Roloff, 1996; Rosolem et al., 1999). Independente da distribuição espacial, a compactação cria um ambiente desfavorável para o crescimento das plantas, associado com o aumento da RP, a qual depende da umidade do solo (Busscher et al., 1997). As indicações são de que solos mais compactados apresentam maior sensibilidade à elevação da RP com o secamento do solo (Tormena et al., 1998a). Valores de RP acima de 2,0 MPa têm sido comumente associados como impeditivos para o crescimento das raízes das plantas (Taylor et al., 1966).

A compreensão e a quantificação do impacto dos sistemas de preparo do solo na sua qualidade física são fundamentais no desenvolvimento de sistemas agrícolas sustentáveis, em especial para a cultura da mandioca. Desta forma, o objetivo deste trabalho foi avaliar o efeito de diferentes sistemas de preparo sobre algumas propriedades físicas de um Latossolo Vermelho distrófico da região noroeste do Estado do Paraná, utilizados consecutivamente, por três anos, para o cultivo da mandioca.

\section{MATERIAL E MÉTODOS}

O experimento foi conduzido em área pertencente à Pinduca Indústria Alimentícia Ltda, no município de Araruna, região noroeste do Estado do Paraná, durante os anos agrícolas 2001/ 2002. O solo em que foi conduzido o experimento é classificado como Latossolo Vermelho distrófico (Embrapa, 1999). A caracterização granulométrica do solo na profundidade de 0-0,30 m forneceu os seguintes valores: areia $=680 \mathrm{~g} \mathrm{~kg}^{-1}$, silte $=10 \mathrm{~g} \mathrm{~kg}^{-1}$ e argila $=310 \mathrm{~g} \mathrm{~kg}^{-1}$, sendo o solo classificado como de classe textural Franco-Argilo-Arenosa (Embrapa, 1999). O clima da região é mesotérmico sem estação seca, com verões quentes, temperatura anual média de $21,5^{\circ} \mathrm{C}$, 
precipitação média anual de 1.617 mm e umidade relativa média anual de 62\% (Godoy et al., 1976).

Os tratamentos utilizados constituíram-se de três sistemas de preparo de solo: Plantio Direto - PD (com revolvimento apenas ao longo das linhas de plantio); Preparo Mínimo PM (escarificação a 0,30 m, seguido de gradagem niveladora); e Preparo Convencional - PC (aração, com arado de aiveca, a uma profundidade entre $0,25-0,30 \mathrm{~m}$, seguido de gradagem niveladora). $\mathrm{O}$ delineamento experimental utilizado foi o de blocos completos casualizados, com oito repetições, perfazendo o total de 24 unidades experimentais. Cada parcela experimental teve área total $147 \mathrm{~m}^{2}(7 \times 21 \mathrm{~m})$ e área útil de $30 \mathrm{~m}^{2}$ ( $2 \times 15 \mathrm{~m})$. O espaçamento utilizado para o plantio da cultura da mandioca foi de $1,0 \mathrm{~m}$ entre linhas e $0,80 \mathrm{~m}$ entre plantas obtendo-se, desta maneira, uma população de 12.500 plantas/ha. Tanto as parcelas quanto os blocos foram espaçados em $1 \mathrm{~m}$. A variedade de mandioca utilizada foi a 'Fécula Branca'.

A implantação do experimento iniciou-se com o plantio de aveia preta (Avena strigosa, Schreb), em abril de 1999 (safra 1999/2000). Quando da floração plena da aveia, a mesma passou por um processo de rolagem por meio de um "rolo-faca". Este procedimento foi realizado também antes dos segundo e terceiro cultivos de mandioca nas safras 2000/2001 e 2001/2002, respectivamente. O plantio da cultura da mandioca foi realizado em agosto-setembro de cada ano.

Para a determinação das propriedades físicas do solo, em julho de 2002 foram retiradas amostras com estrutura indeformada (uma por parcela experimental), por meio de amostrador e anéis com volume de $100 \mathrm{~cm}^{3}$, no centro das camadas de 0-0,15 e 0,15-0,30 m de profundidade. Após a coleta, as amostras foram acondicionadas em sacos plásticos e mantidas sob temperatura de $\pm 5{ }^{\circ} \mathrm{C}$, até serem processadas. A Ds foi determinada de acordo com Blake \& Hartge (1986). Para a determinação da porosidade do solo, as amostras indeformadas foram saturadas por 48 horas em bandeja com água, até dois terços da altura do anel. Após o período de saturação foram obtidas as massas das amostras saturadas e, em seguida, as mesmas foram drenadas na tensão equivalente a 0,006 MPa, utilizando-se uma mesa de tensão. A partir dos valores de retenção de água das amostras calculou-se o volume de macroporos drenados na tensão de 0,006 MPa (Mac), a microporosidade (Mic) e a porosidade total do solo (Pt) de acordo com Embrapa (1997).

A RP foi medida conforme Tormena \& Roloff (1996) e os dados foram obtidos a cada $0,05 \mathrm{~m}$ até $0,40 \mathrm{~m}$ de profundidade, utilizando-se um penetrômetro com anel dinamométrico, com diâmetro de base do cone de $10 \mathrm{~mm}$, além de realizadas as determinações de RP em dois períodos do crescimento da cultura (janeiro de 2002 e junho de 2002), equivalentes a diferentes condições de umidade do solo. Para o solo da área experimental, a umidade do solo na capacidade de campo (CC) conforme Watanabe (2001) é de $0,16 \mathrm{~kg} \mathrm{~kg}^{-1}$. Em cada parcela experimental foram obtidas três medidas de RP e um valor médio de cada profundidade foi utilizado para representar a parcela individualmente. $\mathrm{Na}$ amostragem realizada em janeiro de 2002 determinou-se a umidade do solo em cada parcela experimental, em intervalos de $0,10 \mathrm{~m}$ até $0,40 \mathrm{~m}$ de profundidade.
Para as variáveis Ds e aquelas relativas à porosidade do solo, a análise dos dados foi feita conforme os procedimentos descritos por Hatcher \& Stepanski (1997). Constatando-se efeito significativo da interação entre tratamento e profundidade amostrada $(p<0,05)$ procederam-se aos desdobramentos necessários e se aplicou o teste de Tukey $(p<0,05)$ para a comparação das médias. Os dados de RP foram avaliados utilizando-se um valor médio em cada profundidade e o erro padrão da média foi usado como critério para se proceder à avaliação dos tratamentos em cada profundidade amostrada. Adotou-se o critério de Gravetter \& Wallnau (1995) para diferenciar estatisticamente os tratamentos, indicado pela ausência de sobreposição dos limites superior e inferior dos valores da média \pm erro padrão. As análises estatísticas foram conduzidas utilizando-se o programa estatístico SAS (SAS Institute, 1999).

\section{RESULTADOS E DISCUSSÃO}

Os resultados da avaliação da Ds nas profundidades de $0-0,15$ e $0,15-0,30$ m estão apresentados na Figura 1 . Na profundidade de $0-0,15 \mathrm{~m}$ os sistemas de preparo proporcionaram modificações estatisticamente significativas na Ds $(p<0,05)$ sendo que os maiores valores foram encontrados nos sistemas PM e PD. Resultados similares também foram constatados por outros autores ao compararem diferentes sistemas de preparo do solo (De Maria et al., 1999; Stone \& Silveira, 2001). Os maiores valores de Ds se devem ao tráfego cumulativo de máquinas associado à reduzida mobilização do solo (Tormena et al., 1998b). No PC os menores valores de Ds podem ser atribuídos ao revolvimento do solo e à incorporação dos resíduos culturais.

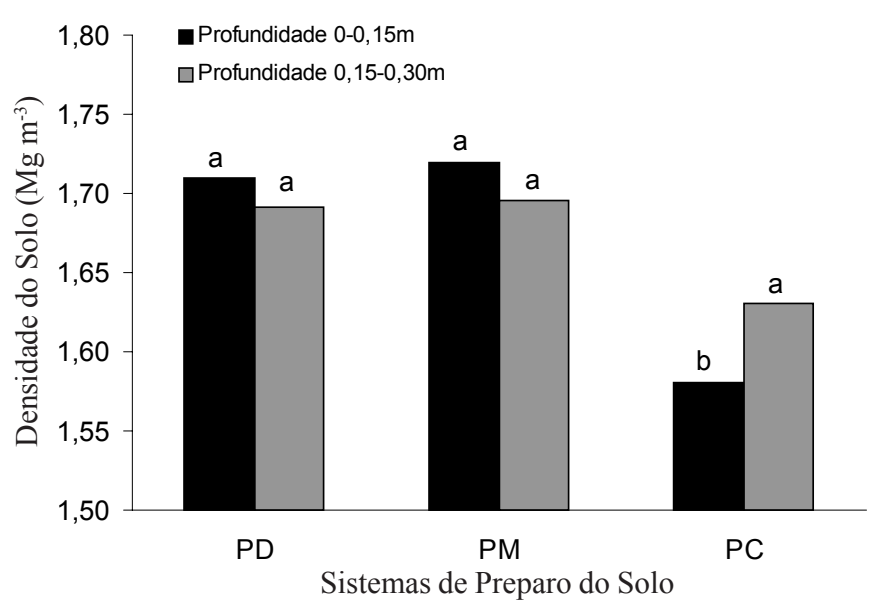

Obs.Para a mesma profundidade, as letras distintas indicam os efeitos significativos pelo teste de Tukey $(\mathrm{p}<0,05)$

Figura 1. Valores da densidade do solo nos diferentes sistemas de preparo para as profundidades $0-0,15 \mathrm{~m}$ e $0,15-0,30 \mathrm{~m}$

Para a profundidade de $0,15-0,30 \mathrm{~m}$ não foram constatadas diferenças estatisticamente significativas entre os sistemas de preparo para a variável Ds (Figura 1). Estes resultados podem estar associados ao confinamento superficial das cargas aplicadas ao solo pelas máquinas, de modo que os efeitos dos tratamentos na compactação do solo ficaram restritos à camada 
superficial, como indicado por Tormena et al. (1998b). Apesar da ausência de diferenças, os resultados indicam tendência do PD e PM apresentarem maiores valores de Ds em relação ao PC, corroborando com os resultados obtidos por Oliveira et al. (2001).

Os resultados da avaliação da porosidade do solo, expressos em valores de Mac, Mic e Pt, para a profundidade de 0-0,15 $\mathrm{m}$, estão apresentados na Figura 2A. Os resultados apresentados mostram que PM e PD promoveram alterações significativas na Pt e Mac, quando comparados com o PC, cujos resultados estão em conformidade com van Ouwerkek \& Boone (1970) indicando que sistemas com menor mobilização do solo, além de reduzir a porosidade total mudam a distribuição do tamanho dos poros, com redução dos poros de maior tamanho. A utilização do arado de aiveca com intensa mobilização do solo no tratamento $\mathrm{PC}$, proporcionou maiores valores de Mac e Pt $(p<0,001)$ em comparação com o PD e o PM. Os maiores valores de Mac obtidos em PC comparados com PD são devidos, provavelmente, à persistência dos efeitos da mobilização do solo no PC e à ausência de revolvimento do solo e à movimentação de máquinas e implementos agrícolas no PD, como atestam os resultados obtidos por Stone \& Silveira (2001). Os resultados indicam, também, que nos sistemas PM e PD ocorreu, com o aumento da Ds, diminuição em Mac, o que está de acordo com os resultados obtidos por Tormena et al. (1998b) e Watanabe et al. (2001). De acordo com Tollner et al.
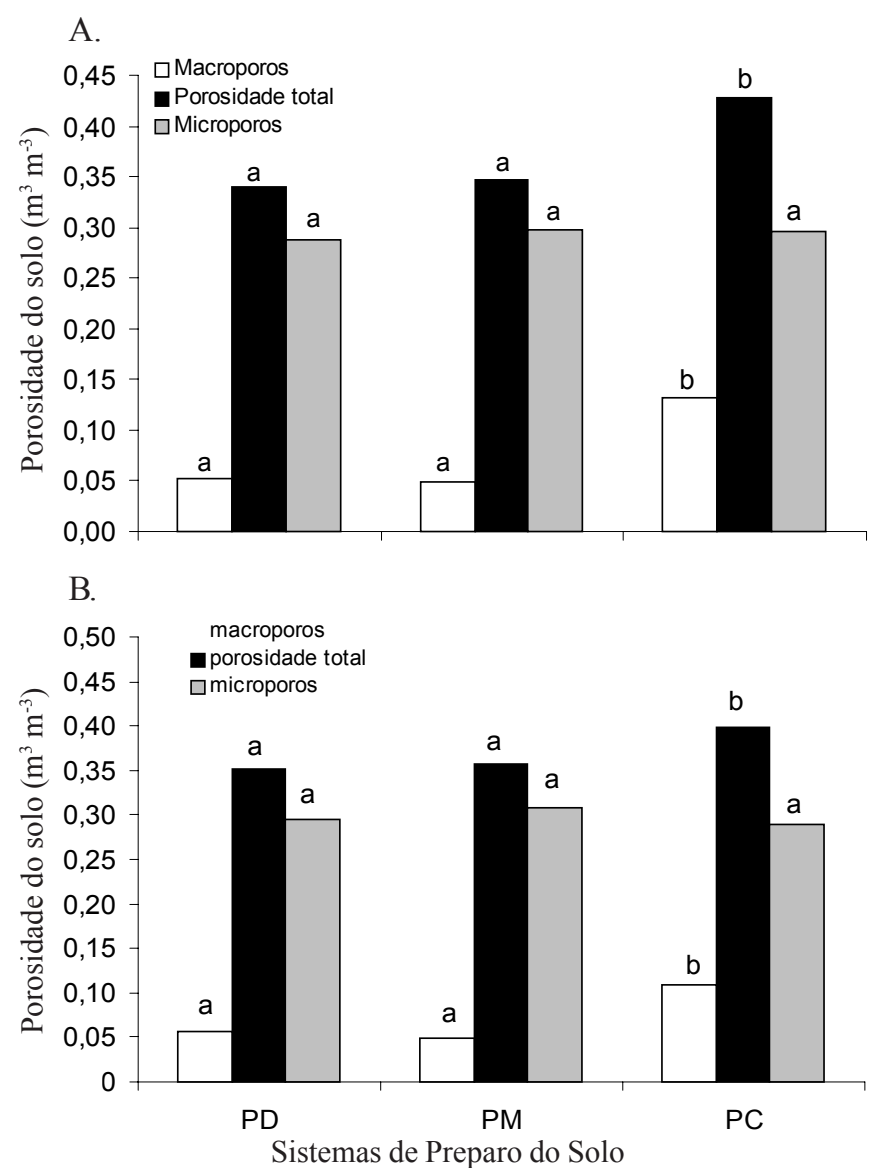

Obs.Para cada variável, letras minúsculas distintas indicam que os tratamentos foram significativamente diferentes pelo teste de Tukey $(\mathrm{p}<0,05)$

Figura 2. Valores de porosidade do solo nos diferentes sistemas de preparo para as profundidades de $0-0,15 \mathrm{~m}(\mathrm{~A}) \mathrm{e} 0,15-0,30 \mathrm{~m}$ (B)
(1984), o revolvimento do solo aumenta o número de poros drenáveis, constatado neste trabalho pelo aumento no volume de macroporos no PC. Desta forma, sob PC o solo poderia drenar mais rapidamente que em PD e PM, com influência na disponibilidade de água às plantas e na aeração do solo, em períodos úmidos mais prolongados. Condições de aeração deficiente podem ter fundamental importância para a sanidade das raízes da cultura da mandioca, em especial para cultivares mais sensíveis a doenças. De acordo com a Figura 2A, condições potencialmente restritivas de aeração podem ocorrer no PD e PM, cujo valor médio de Mac é da ordem de 5\%.

Os valores de Mic não diferiram entre os tratamentos (Figura 2A). Este comportamento corrobora com as constatações de Silva \& Kay (1997) de que a Mic é muito pouco influenciada pelo aumento na Ds, o que justifica a similaridade de Mic entre os tratamentos.

Para a camada de $0,15-0,30 \mathrm{~m}$, os resultados estão apresentados na Figura 2B. Nesta profundidade, os valores médios de Pt e Mac apresentaram o mesmo comportamento da camada anterior. Também não se constatou diferença significativa para a variável Mic $(\mathrm{p}>0,05)$; para as variáveis Mac e Pt, os valores em PC foram significativamente superiores a PD e PM $(\mathrm{p}<0,05)$, resultados esses devidos aos efeitos do revolvimento do solo pelo arado e à sua preservação após o preparo. Resultados similares foram obtidos por Stone \& Silveira (2001). No PM, processos que levaram à recompactação do solo após a escarificação, podem ter contribuído para os resultados, fato confirmado pelos resultados obtidos por Busscher et al. (2002).

Em geral, os resultados indicam que Pt e Mac foram mais sensíveis que a Ds e Mic aos efeitos dos tratamentos. Neste sentido, em profundidade os tratamentos promoveram alterações que foram retratadas pela porosidade (Pt e Mac); no entanto, estas alterações não foram suficientes para alterar a Ds e a Mic. A redução de Ds em PC foi refletida estatisticamente em maiores valores de Mac e Pt como evidenciado por Tormena et al. (1998b) cujos resultados confirmam que os macroporos são a classe de poros menos estáveis e sofrem colapso quando submetidos aos estresses aplicados pelos sistemas de preparo e tráfego; neste caso, independente da profundidade em que foi feita a avaliação.

Os resultados da avaliação de RP são apresentados nas Figuras 3A e B. Os resultados de RP obtidos na determinação realizada em janeiro de 2002 estão apresentados na Figura 3A e os respectivos valores de umidade do solo encontram-se na Figura 4. Nesta avaliação, a RP foi medida com umidade do solo abaixo da capacidade de campo (CC). Levando-se em conta o valor de $0,16 \mathrm{~kg} \mathrm{~kg}^{-1}$ para a CC (Watanabe, 2001), as umidades aumentaram em profundidade, aproximando-se da CC, principalmente no $\mathrm{PD}$, o que correspondeu a uma redução na $\mathrm{RP}$ com relação às camadas superficiais. No tratamento $\mathrm{PD}$, a umidade foi superior ao PM, que não foi diferente de PC, em todas as profundidades. A umidade em PD foi superior à do PC apenas na profundidade de 0-0,10 m. Apesar de PD apresentar maiores valores de umidade (Figura 4 ) os resultados indicam maiores valores de RP na faixa de 0,05 a 0,25 m em comparação com PC e PM (Figura 3A). Constata-se que a maior umidade no PD não correspondeu a uma redução na RP: até a $0,25 \mathrm{~m}$ de 
profundidade a RP foi superior no PD em relação ao PM e PC (Figura 3A). A partir daí, os valores de RP no PD foram significativamente menores que os do PC e PM, o que pode estar associado à maior umidade no $\mathrm{PD}$ e ao efeito residual do revolvimento pelos órgãos ativos dos implementos no PC e no PM.
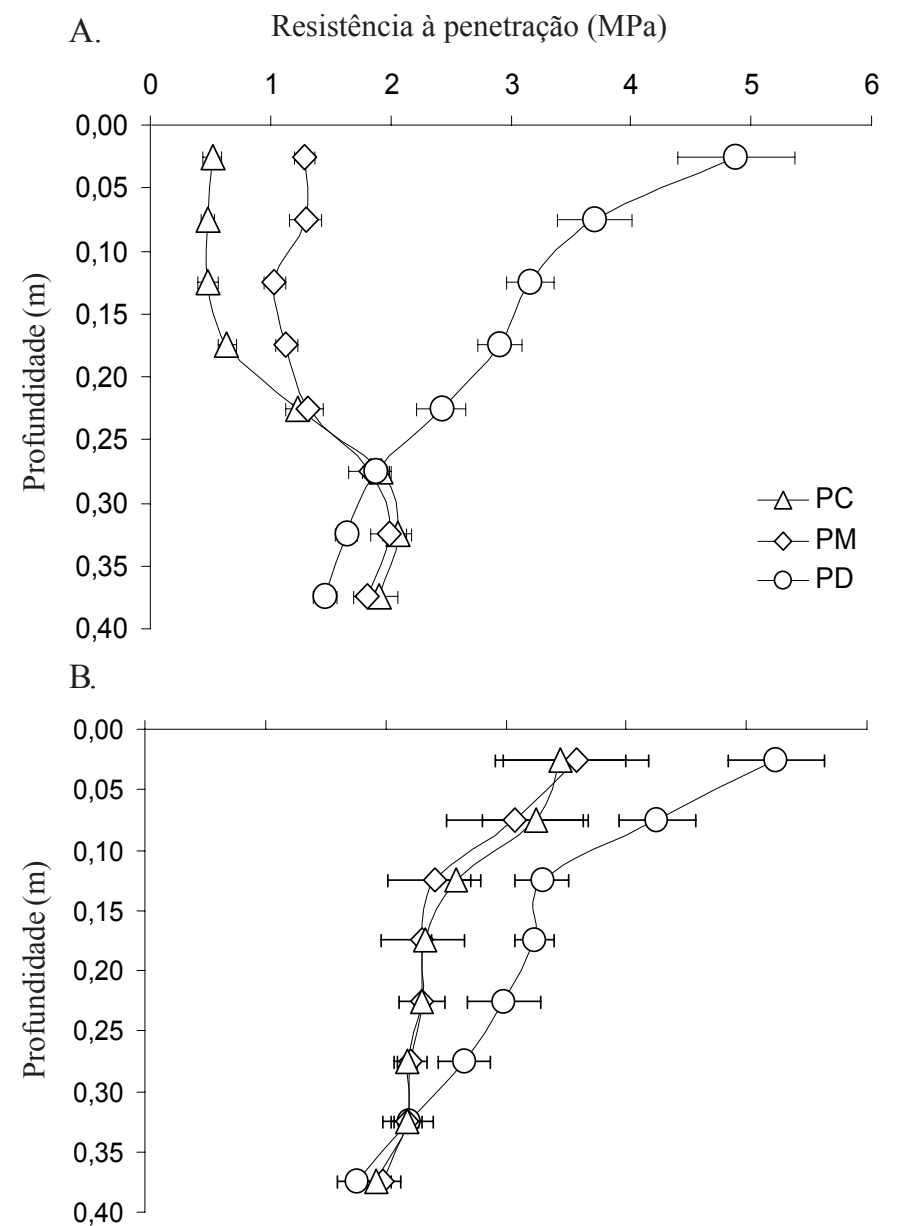

As barras indicam os valores de erro padrão da média e sua sobreposição denota a ausência de diferenças entre as médias dos tratamentos

Figura 3. Resistência do solo à penetração na amostragem realizada em janeiro de 2002 (A) e junho de 2002 (B) nos diferentes sistemas de preparo

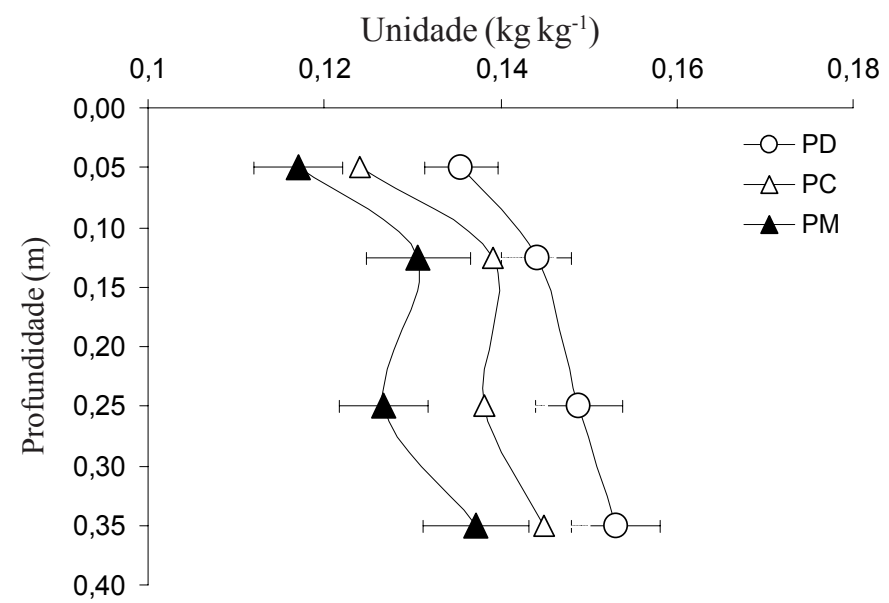

As barras indicam os valores do erro padrão da média e sua sobreposição denota a ausência de diferenças entre as médias dos tratamentos

Figura 4. Umidade do solo na amostragem realizada em janeiro de 2002 nos diferentes sistemas de preparo
Estes resultados estão relacionados com a maior Ds no PD, implicando em maior resistência ao deslocamento das partículas do solo, por ocasião da medida de RP. No PM, planos de fraqueza gerados no processo de escarificação condicionam que os valores de RP sejam substancialmente reduzidos. Tais interações da RP com as condições estruturais do solo também têm sido constatadas por outros autores (Tormena et al., 1998a, Imhoff et al., 2000). Desta forma, os resultados sugerem que, para determinada condição de umidade, no PD ocorrem os maiores valores de RP, em relação ao $\mathrm{PC}$ e ao $\mathrm{PM}$, predispondo as culturas a níveis impeditivos de RP, ao longo do período vegetativo e durante a formação e crescimento das raízes tuberosas da cultura da mandioca.

Os valores de RP constatados no PD atingiram valores altamente impeditivos ao crescimento das plantas ( $\mathrm{RP}>2,0$ $\mathrm{MPa}$ ) até $0,25 \mathrm{~m}$ de profundidade, em comparação com o PC e $\mathrm{PM}$, resultados estes devidos à maior compactação do solo no PD em relação ao PC e PM, indicada pelas variáveis Ds, Pt e Mac (Figuras 1 e 2), reflexos do tráfego cumulativo de máquinas e da ausência de movimentação do solo no PD. Em termos da qualidade dos solos para o crescimento das plantas, elevados valores de RP no PD podem constituir-se em impedimentos para as culturas. As razões, de acordo com Passioura (1991) decorrem do fato da RP afetar diretamente o crescimento das raízes e, conseqüentemente, a absorção de água e nutrientes, além de estabelecer mecanismos não-hidráulicos de controle da taxa de condutância estomatal, da fotossíntese, e da produção de fotoassimilados.

Os resultados obtidos com a RP neste trabalho estão em conformidade com os obtidos por De Maria et al. (1999) e Tavares Filho et al. (2001), constatando que a redução de revolvimento afeta negativamente os valores de algumas variáveis que expressam a qualidade física do solo. As maiores diferenças entre os sistemas de preparo estão concentradas nas camadas superficiais do solo, refletindo suas ações e interações com os processos da interface solo-planta-atmosfera.

Os dados de RP da avaliação realizada em junho de 2002, são apresentados na Figura 3B. Nesta amostragem não foi determinada a umidade do solo, mas as observações de campo indicavam uma condição de umidade inferior à amostragem realizada em janeiro de 2002; no entanto, nesta avaliação os resultados indicam que os valores de RP podem ser considerados impeditivos em todos os tratamentos ( $\mathrm{RP}=2,0$ $\mathrm{MPa}$ ) até $0,30 \mathrm{~m}$ de profundidade. A tendência observada na coleta anterior se manteve até $0,25 \mathrm{~m}$ de profundidade, ou seja, a RP foi significativamente maior no PD que no PC e PM, denotando a manutenção das características estruturais determinadas pelos sistemas de preparo do solo. Em profundidade ocorreu redução nos valores de RP em todos os tratamentos, não sendo constatadas diferenças significativas entre eles a partir de $0,30 \mathrm{~m}$. Estes resultados estão em concordância com os obtidos por diversos outros autores (Tormena \& Roloff, 1996; Watanabe, 2001)

Finalmente, constata-se que a mobilização do solo por meio do PC e PM reduziu substancialmente a RP em comparação com o PD. Os resultados de RP sugerem que, no processo de secamento do solo sob PD, ocorre um aumento expressivo da RP. De forma geral, PD apresenta condições potencialmente 
restritivas para o crescimento da cultura, face aos elevados valores de RP e Ds, bem como aos reduzidos valores de Mac. Sugere-se a necessidade de se realizar pesquisas com maior tempo de implantação dos sistemas de preparo, para que uma avaliação mais eficaz possa ser realizada. Além disso, medidas como da curva de resistência do solo podem auxiliar na quantificação do comportamento físico do solo e na determinação dos efeitos dos sistemas de preparo na qualidade física do solo, para o crescimento das plantas, em adição a medidas pontuais de RP, Ds e porosidade do solo.

\section{CONCLUSÕES}

1. Na camada superficial, a Ds foi reduzida sob as condições do PC em comparação com o PD e PM.

2. Nos sistemas PD e PM a Mac e a Pt foram menores que no PC, e seus valores de Mac podem ser considerados restritivos para a aeração do solo.

3. No PD, os valores de RP foram maiores que no PC e PM e estão relacionados com o aumento da Ds.

\section{LITERATURA CITADA}

Blake, G.R.; Hartge, K.H. Bulk density. In: Klute, A.(ed.). Methods of soil analysis: Physical and mineralogical methods. 2 ed. Madison: America Society of Agronomy, 1986. cap.13, p.363-375.

Busscher, W.J.; Bauer, P.J.; Camp, C.R.; Sojka, R.E. Correction of cone index for soil water content differences in a coastal plain soil. Soil Tillage Research, Amsterdam, v.43, p.205217, 1997.

Busscher, W.J.; Bauer, P.J.; Frederick, J.R. Recompaction of a coastal loamy sand after deep tillage as a function of subsequent cumulative rainfall. Soil Tillage Research, Amsterdam, v.68, n.1, p.49-57, 2002.

Cadavid, L.F.; El-Sharkawy, M.A.; Acosta, A.; Sánchez, T. Longterm effects of mulch, fertilization and tillage on cassava grown in sandy soils in northern Colombia. Field Crops Research, Amsterdam, v.57, p.45-56, 1998.

Cardoso, A.; Potter, R.; Dedecek, R.A. Estudo comparativo da degradação de solos pelo uso agrícola no Noroeste do Estado do Paraná. Pesquisa Agropecuária Brasileira, Brasília, v.27, p.349-353, 1992.

Carvalho, A.P. Solos do arenito caiuá. In: Pereira, P.P.; Ferreira, M.E.; Pessoa da Cruz, M.C. (eds). Solos altamente susceptíveis à erosão. Jaboticabal: UNESP/SBCS, 1994, p.39-49, Cap. 2

Davies, W.J.; Zangh, J. Root signals and the regulation of growth and development of plants in drying soil. Annual Review of Plant Physiology and Plant Molecular Biology, Palo Alto, v.42, p.55-76, 1991.

De Maria, I.C.; Castro, O.M.; Souza Dias, H. Atributos físicos do solo e crescimento radicular de soja em Latossolo Roxo sob diferentes métodos de preparo do solo. Revista Brasileira de Ciência do Solo, Viçosa, v. 23, p. 703-709, 1999.

EMBRAPA. Empresa Brasileira de Pesquisa Agropecuária. Centro Nacional de Pesquisa de Solos. Manual de métodos de análise de solo. 2.ed. Rio de Janeiro, 1997. 212p.
EMBRAPA. Empresa Brasileira de Pesquisa Agropecuária. Sistema brasileiro de classificação de solos. Rio de Janeiro, 1999, $412 \mathrm{p}$.

Fidalski, J. Fertilidade do solo sob pastagens, lavouras anuais e permanentes na região Noroeste do Paraná. Revista Unimar, Maringá, v. 19, p. 853-861, 1997.

Godoy, H.; Correa, A.R.; Santos, D. Clima do Paraná. In: Fundação Instituto Agronômico do Paraná. Manual Agropecuário para o Paraná. Londrina 1976. p.17-36.

Gravetter, F.J.; Wallnau, L.B. Statistics for the behavioral sciences. 2.ed. St. Paul: West Publishing, 1995. 429p.

Groxko, M. Mandioca. In: Acompanhamento da situação agropecuária no Paraná. Curitiba: SEAB/DERAL, v.23, n.9, p.89-95, 1997.

Hatcher, L.; Stepanski, E.J. A step-by-step approach to using the SAS system for univariate and multivariate statistics. Cary: NC: SAS Institute Inc., 1997. 552p.

Howeler, R.H.; Ezumah, H.C.; Midmore, D.J. Tillage systems for root and tuber crops in the tropics. Soil and Tillage Research, Amsterdam, v.27, p.211-240, 1993.

Imhoff, S.; Da Silva, A. P.; Tormena, C. A. Aplicações da curva de resistência no controle da qualidade física de um solo sob pastagem. Pesquisa Agropecuária Brasileira, Brasília, v.35, p.1493-1500, 2000.

Kay, B.D. Rates of changes of soil structure under different cropping systems. Advances in Soil Science, London, v.12, p.1-51, 1990 .

Kay, B.D.; Angers, D.A. Soil structure. In: Summer, M.E. (ed). Handbook of soil science. New York: CRC Press, p.A229A275, 2000.

Klepker, D.; Anghinoni, I. Características físicas e químicas do solo afetadas por métodos de preparo e modos de adubação. Revista Brasileira de Ciência do Solo, Viçosa, v.19, p.395401, 1995.

Oliveira, J.O.A.P.; Vidigal Filho, P.S.; Tormena, C.A.; Pequeno, M.G.; Scapim, C.A.; Muniz, A.S.; Sagrilo, E. Influência de sistemas de preparo do solo na produtividade da mandioca (Manihot esculenta, Crantz). Revista Brasileira de Ciência do Solo, Viçosa, v.25, p.443-450, 2001.

Passioura, J.B. Soil structure and plant growth. Australian Journal of Soil Research, Sydney, v.29, n.6, p.717-728, 1991.

Rosolem, C.A.; Fernandez, E.M.; Andreotti, M.; Crusciol, C.A.C. Crescimento radicular de plântulas de milho afetado pela resistência do solo à penetração. Pesquisa Agropecuária Brasileira, Brasília, v. 34, n. 5, p. 821-828, 1999.

SAS - Statistical Analysis System Institute. SAS/STAT Procedure guide for personal computers. 5. ed. Cary, NC: SAS Inst. $1999.334 \mathrm{p}$.

Silva, A.P.; Kay, B.D. Estimating the least limiting water range of soils from properties and management. Soil Science Society of America Journal, Madison, v.61, p.877-883, 1997.

Souza, L.S. Manejo e conservação do solo para a cultura da mandioca. In: Curso Intensivo Nacional de Mandioca, 6, 1986, Cruz das Almas: EMBRAPA/CNPMF, 1986. 40p. 
Stone, L.F.; Silveira, P.M. Efeitos do sistema de preparo e da rotação de culturas na porosidade e densidade do solo. Revista Brasileira de Ciência do Solo, Viçosa, v. 25, p. 395401, 2001.

Tavares Filho, J.; Barbosa, G.M.C.; Guimarães, M.F.; Fonseca, I.C.B. Resistência do solo à penetração e desenvolvimento do sistema radicular do milho (Zea mays) sob diferentes sistemas de manejo em um Latossolo Roxo. Revista Brasileira de Ciência do Solo, Viçosa, v.25, p.725-730, 2001.

Taylor, H.M.; Roberson, G.M.; Parker Jr, J.J. Soil strength-root penetration relations to medium to coarse-textured soil materials. Soil Science, Baltimore, v.102, p.18-22, 1966.

Tollner, E.W.; Hargrove, W.L.; Langdale, G.W. Influence of conventional and no-tillage practices on soil physical properties in the Southern Piedmont. Journal of Soil and Water Conservation, Ankeny, v.39, n.1, p.73-76, 1984.
Tormena, C.A.; Roloff, G. Dinâmica da resistência à penetração de um solo sob plantio direto. Revista Brasileira de Ciência do Solo, Viçosa, v.20, p.333-339, 1996.

Tormena, C.A.; Roloff, G.; Sá, J. C. M. Propriedades físicas do solo sob plantio direto influenciado por calagem, preparo inicial e tráfego. Revista Brasileira de Ciência do Solo, Viçosa, v. 22, p. 301-309, 1998b.

Tormena, C.A.; Silva, A.P.; Libardi, P.L. Caracterização do intervalo hídrico ótimo de um Latossolo Roxo sob plantio direto. Revista Brasileira de Ciência do Solo, Viçosa, v. 22, p.573-581, 1998a.

van Ouwerkerk, C.; Boone, F.R. Soil-physical aspects of zerotillage experiments. Netherlands Journal of Agricultural Science, The Hague, v.18, p.247-261, 1970.

Watanabe, S.H. Caracterização da qualidade física de um Latossolo Vermelho distrófico sob diferentes sistemas de preparo. Maringá: UEM, 2001. 69p. Dissertação Mestrado 